psychopraxis. neuropraxis $2015 \cdot 18: 31$ DOI 10.1007/s00739-015-0242-5

Online publiziert: 12. März 2015

○) Springer-Verlag Wien 2015

Gabriele Sachs

Universitätsklinik für Psychiatrie und Psychotherapie, Medizinische Universität Wien, Wien, Österreich

\title{
Differenzierte Diagnostik depressiver Störungen
}

affektiver Störungen, dass Asenapin eine Überlegenheit gegenüber Olanzapin oder Plazebo aufzeigt, je „gemischter“ eine manische Episode ist. In den USA hat Asenapin neben der Manie- und Schizophrenieindikation bereits eine Zulassung spezifisch für die akute Behandlung gemischter Episoden, die mit Bipolar-I-Störungen gemäß DSM assoziiert sind [4].

Nicht zuletzt auf dem Boden dieser Überlegungen erscheint eine genauere Auseinandersetzung mit den Zusatzcodierungen der Major Depression sinnvoll. Unter anderen informativen Artikeln zu verschiedenen neuropsychiatrischen Erkrankungen werden Sie in diesem Heft der psychopraxis.neuropraxis einen Beitrag finden, der Sie über die Major Depression mit der Zusatzcodierung „mit gemischten Merkmalen“ informieren möchte und der die modernen Konzepte des DSM 5 mit älteren Vorstellungen in der deutschsprachigen Psychiatrie vergleicht.

Viel Vergnügen bei der Lektüre dieses Heftes wünscht Ihnen

Ihre

\section{Gabriele Sachs}

\section{Korrespondenzadresse}

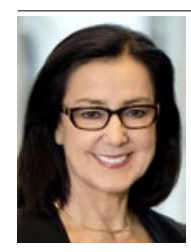

Ao. Univ.-Prof. DDr. G. Sachs Universitätsklinik für Psychiatrie und Psychotherapie Medizinische Universität Wien Währinger Gürtel 18-20 1090 Wien gabriele.sachs@meduniwien. ac.at

\section{Einhaltung ethischer Richtlinien}

Interessenkonflikt. G. Sachs gibt an, dass kein Interessenkonflikt besteht.

\section{Literatur}

1. Mathers CD, Loncar D (2006) Projections of global mortality and burden of disease from 2002 to 2030. PLoS Med 3:e442

2. American Psychiatric Association (2015) Diagnostisches und Statistisches Manual Psychischer Störungen DSM-5 ${ }^{\circledR}$. Deutsche Ausgabe. Falkai P, Wittchen HU (Hrsg) Hogrefe, Göttingen

3. Mclntyre RS, Tohen M, Berk M, Zhao J, Weiller E (2013) DSM-5 mixed specifier for manic episodes: evaluating the effect of depressive features on severity and treatment outcome using asenapine clinical trial data. J Affect Disord 150(2):378-383

4. http://www.accessdata.fda.gov/drugsatfda_docs/ label/2011/022117s010lbl.pdf Zugegriffen: 23 . Feb. 2015

Es ist zu erwarten, dass diese Zusatzcodierungen auch bei den Zulassungen für neue Psychopharmaka eine zunehmende Rolle spielen werden. So beschreiben McIntyre et al. [3] für den manischen Pol 